\title{
Antifungal Activity of Ethanol-extracted Bixa orellana (L) (Achiote) on Candida albicans, at Six Different Concentrations
}

\author{
Lucia Poma-Castillo ${ }^{1}$, María Espinoza-Poma ${ }^{1}$, Franco Mauricio ${ }^{1}$, Cesar Mauricio-Vilchez ${ }^{2}$, Daniel Alvítez-Temoche ${ }^{2}$, \\ Frank Mayta-Tovalino 3
}

\begin{abstract}
Aim: The aim of the study was to determine the antifungal activity of six different concentrations of the ethanol extract of Bixa orellana ( $L$ ) (Achiote) on the strains of Candida albicans (ATCC 90028).

Materials and methods: The ethanol extract of Bixa orellana (L) (BEE) was prepared in six different concentrations (5\%, $10 \%, 20 \%, 40 \%, 60 \%$, and $100 \%$ ), which were confronted with the positive control group (Nystatin 100,000 Ul/mL). The total sample consisted of $n=174$ wells inoculated with each concentration evaluated in the Petri dishes. The cultivation procedure was carried out at a temperature of $37^{\circ} \mathrm{C}$, and then the $6 \mathrm{~mm}$ diameter wells on which the sowing of the Candida albicans in suspension was carried out. Subsequently, the BEE (Achiote) was inoculated in all the concentrations mentioned in the same way for Nystatin; finally, the inhibition halos were evaluated by the Kirby-Bauer method at the scheduled times of 24,48 , and 72 hours.

Results: The highest antifungal activity was found at 24 hours and this increased directly proportional to the concentration of BEE (5\%, $10 \%$, $20 \%, 40 \%, 60 \%$, and 100\%): $11 \pm 0,11 \pm 0,13.1 \pm 1.3,17.2 \pm 0.8,19.1 \pm 1.0$, and $21.4 \pm 1.3 \mathrm{~mm}$, respectively. However, there was no statistically significant difference when compared with Nystatin, at $p>0.05$

Conclusion: It was shown that 5\%, 10\%, 20\%, 40\%,60\%, and 100\% BEE had an antifungal effect against Candida albicans. However, there were no statistically significant differences against the effect of Nystatin at 100,000 IU/mL, who has an inhibitory effect greater than BEE, with a significance level of $p<0.05$.

Clinical significance: This article describes the antifungal activity and the possible impact on dentistry that the use of Bixa orellana (L) may have because according to our results it had an antifungal effect against strains of Candida albicans. This opens a great line of research to potentially use this natural resource in dentistry.

Keywords: Achiote, Antifungal activity, Bixa orellana (L), Candida albicans.

The Journal of Contemporary Dental Practice (2019): 10.5005/jp-journals-10024-2672
\end{abstract}

\section{INTRODUCTION}

Candida albicans is found in the oral cavity between $25 \%$ and $50 \%$ in healthy people (adults or children). This pathogen is an opportunistic fungus with the ability to subsist as a host, adhering to the cells of the oral mucosa to subsequently cause the development of certain diseases of the oral cavity. The use of dental prosthesis is usually associated with the presence of candidiasis or moniliasis, which is one of the most frequent infections in the mouth, especially in patients with dental prostheses; candidiasis is persistent because it usually adheres to both the host and the prosthesis. There are different risk factors that can trigger the onset of the disease, such as lack of hygiene, systemic factors, immunosuppression, and prolonged pharmacological treatment, etc. ${ }^{1-3}$

According to scientific literature, the prevalence of the appearance of Candida albicans in patients with dentures is above $60 \%$, with this fungus regularly located in the mucosa of the hard and soft palate below the prosthesis. Candidiasis usually appears in the internal part of the dental prosthesis, and being directly in contact with the mucosa of the palate tends to induce soft tissues to swell owing to the colonization of this fungus. Most patients do not know how to properly maintain and care for their prosthesis. Daily cleaning is important to avoid the accumulation of plaque, calculus, and pigmentations because these food-remains contribute to the generation of irritations and infections, potentially leading to
${ }^{1}$ Doctoral Program in Dentistry, Faculty of Dentistry, Universidad Nacional Federico Villarreal, Lima, Peru

${ }^{2}$ Faculty of Dentistry, Universidad Nacional Federico Villarreal, Lima, Peru

${ }^{3}$ Postgrade Department, School of Stomatology, Universidad Científica del Sur, Lima, Peru

Corresponding Author: Frank Mayta-Tovalino, Postgrade Department, School of Stomatology, Universidad Cientifica del Sur, Lima, Peru, Phone: +51 1 6106400, e-mail: fmaytat@ucientifica.edu.pe

How to cite this article: Poma-Castillo L, Espinoza-Poma M, Mauricio F, et al. Antifungal Activity of Ethanol-extracted Bixa orellana (L) (Achiote) on Candida albicans, at Six Different Concentrations. J Contemp Dent Pract 2019;20(10):1159-1163.

Source of support: Nil

Conflict of interest: None

the appearance of subprosthetic stomatitis in the adjacent mucosa. The mechanical method using brushes with toothpastes is the most popular method to remove plaque from dentures. However, there is evidence that using this method alone is not enough to eliminate the microbial flora from the bases of the dental prosthesis; so it is

(0) The Author(s). 2019 Open Access This article is distributed under the terms of the Creative Commons Attribution 4.0 International License (https://creativecommons. org/licenses/by-nc/4.0/), which permits unrestricted use, distribution, and non-commercial reproduction in any medium, provided you give appropriate credit to the original author(s) and the source, provide a link to the Creative Commons license, and indicate if changes were made. The Creative Commons Public Domain Dedication waiver (http://creativecommons.org/publicdomain/zero/1.0/) applies to the data made available in this article, unless otherwise stated. 
recommended to use certain antimicrobial substances that help reduce these microorganisms. ${ }^{4-7}$

In cases of subprosthetic stomatitis, treatment is usually based on the elimination of local elements; therefore, it is suggested to remove the prosthesis for intermittent times, perform the corresponding hygiene. In phytotherapy, there are several plants used as treatment elements for various diseases, including the fruits of the tropical shrub Bixa orellana $(\mathrm{L})$, which is composed of different carotenes (bixin, norbixin). ${ }^{8,9}$

Bixa orellana $(\mathrm{L})$ is a plant native to the Peruvian and American Amazon that was cultivated and domesticated since pre-Hispanic times. It is usually a small tree and has a dense foliage with a broad trunk. The most attractive part is its fruits that have a capsular shape, inside which are the deep red seeds. ${ }^{9}$ Currently, the leaves, stems, and seeds of this plant have multiple applications in health sciences (new drugs with medicinal potential) as well as beauty (industrial makeup) and textile industries (natural dye for clothes). In the search to try to find new and possible alternative treatments with medicinal plants, ${ }^{9-11}$ this research proposes to evaluate the antifungal activity of Bixa orellana (L) (Achiote) on Candida albicans, which is a microorganism that is normally found in oral cavity, producing infection called oral candidiasis.

Therefore, the purpose of the study was to evaluate the antifungal activity of BEE (Achiote) in six different concentrations on Candida albicans (ATCC 90028).

\section{Materials and Methods}

The present in vitro study was conducted at the Microbiology Laboratory of the Universidad Nacional Federico Villarreal, Lima, Peru. A total sample of $n=174$ wells were embedded with each of the six concentrations of Bixa orellana (L) $(5 \%, 10 \%, 20 \%, 40 \%, 60 \%$ and $100 \%$ ) was evaluated and contrasted with the group positive control (Nystatin at 100,000 IU/mL). The sample size was calculated using the means' comparison formula using a beta of 0.8 and an alpha of 0.05 using the Stata ${ }^{\oplus} 12.0$ statistical software. Next, the following experimental groups were formed:

Group I: strains of Candida albicans facing BEE 5\%

Group II: strains of Candida albicans facing BEE 10\%

Group III: strains of Candida albicans facing BEE 20\%

Group IV: strains of Candida albicans facing BEE $40 \%$

Group V: strains of Candida albicans facing BEE $60 \%$

Group VI: strains of Candida albicans facing BEE 100\%

\section{Obtaining the Extract of Bixa orellana (L) (Achiote)}

With the collaboration of specialist in botany, the Bixa orellana $(\mathrm{L})$ (Achiote) was obtained from the district of Chanchamayo, located in the department of Junín-Peru, whose coordinates are $10^{\circ} 58^{\prime} 39^{\prime \prime} \mathrm{S}$ and $75^{\circ} 10^{\prime} 07^{\prime} \mathrm{W}$ of latitude and longitude at an elevation of 1,242.00 meters above the sea level. The exact identification of the species obtained as the Bixa orellana (L) (Achiote) plant (Fig. 1) was made. BEE was elaborated in Universidad Nacional Mayor de San Marcos, Lima-Peru, Faculty of Pharmacy and Biochemistry, CENPROFARMA Analytical Control Center-CCA. Approximately $0.5 \mathrm{~kg}$ of the leaves of the plant were obtained and then they were placed inside the oven at a temperature of $48^{\circ} \mathrm{C}$ for 8 hours in order to eliminate all types of moisture. Then the dried leaves were ground until they were completely pulverized. About $250 \mathrm{~g}$ of the powdered leaves were weighed and macerated in an amber flask for a period of 5-10 days with 3 liters of ethanol at $96^{\circ}$, then the residue was filtered with Whatman No. 40 filter paper and placed in the oven for a total



Fig. 1: Bixa orellana (L) (Achiote)

evaporation of ethanol at $48^{\circ} \mathrm{C}$. Approximately $0.9 \mathrm{~g}$ were obtained for every $10 \mathrm{~mL}$ of the BEE sample; after 10 days of evaporation, it was deposited in sterile vials for later use. ${ }^{11-13}$

\section{Reactivation of the Fungal Strain}

To carry out the reactivation of Candida albicans (ATCC 90028), Sabouraud broth with dextrose was used in this work. A suitable temperature was used for the culture of the strain $\left(37^{\circ} \mathrm{C}\right)$, and then before carrying out the inoculation, the recognition of the strains was carried out with Gram staining for a period of 24 hours. Fortyfour Petri dishes (100 mm $\times 15 \mathrm{~mm}$ ) (4 wells per Petri dish) were used to confront the six different concentrations of BEE. Then, the selected culture medium (Mueller Hinton agar) with a thickness of $6 \mathrm{~mm}$ of the agar per plate was plated. Labeling was performed on the petri dishes for the identification of the six concentrations of Bixa orellana (L) (5\%, 10\%, 20\%, 40\%, 60\%, and 100\%) and Nystatin at $100,000 \mathrm{IU} / \mathrm{mL}$.

\section{Sensitivity Test and Inoculum Preparation}

The agar diffusion method was used by wells and the preparation of wells was carried out with a test tube reaching about $6 \mathrm{~mm}$ in diameter in which the Candida albicans were planted in suspension, on which placed, the extract of Bixa orellana (L) (Achiote), 5\%, 10\%, $20 \%, 40 \%, 60 \%, 100 \%$ and the Nystatin at $100,000 \mathrm{IU} / \mathrm{mL}$ the method used was Kirby-Bauer (Fig. 2). The standardization of the inoculum was carried out considering the turbidity and the seeding in Petri dishes, for which the hyssop was used in the content of the plates with sabouraud agar with dextrose in a uniform way for all the plates with rotations and directing so that all its surface was left covered.

\section{Inoculation of the Active Principle}

On the elaborated wells, approximately $20 \mu \mathrm{L}$ was deposited looking for them to have the same level in all with the help of a calibrated micropipette. The temperature used was $37^{\circ} \mathrm{C}$, where later with the help of a Vernier rule, the measurement of the halos was carried out at 24,48 , and 72 hours respectively, having verified that the medium is correctly sterile.

\section{Statistical Analysis}

For the statistical analysis, the SPSS (version 23) program was used, since the data analyzed in the respective groups did not have a normal distribution; therefore, non-parametric tests were used 

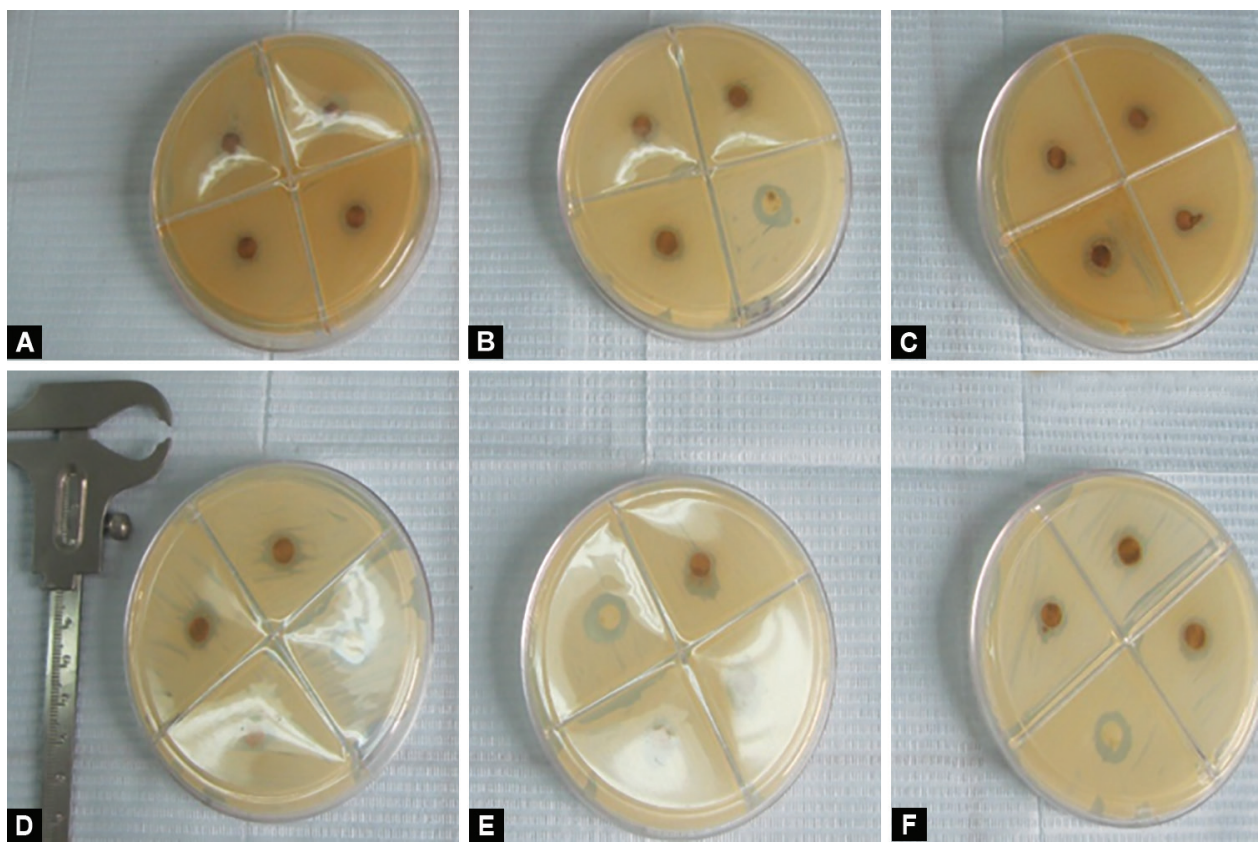

Figs $2 \mathrm{~A}$ to $\mathrm{F}$ : Antifungal confrontation of the six different concentrations $(A=5 \%, B=10 \%, C=20 \%, D=40 \%, E=60 \%$ and $F=100 \%)$ of $B E E$ against Candida albicans strains using the Kirby-Bauer Method

Table 1: Evaluation of the antifungal effect of BEE at different concentrations according to time

\begin{tabular}{llllllll}
\hline & \multicolumn{2}{l}{ 24 hours, mean \pm SD } & \multicolumn{2}{l}{ 48 hours, mean \pm SD } & \multicolumn{2}{l}{ 72 hours, mean \pm SD } & $p$ \\
\hline BEE 5\% & 11.0 & 0.0 & 11.0 & 0.0 & 11.0 & 0.0 & \\
BEE 10\% & 11.0 & 0.0 & 11.0 & 0.1 & 11.0 & 0.0 & 0.340 \\
BEE 20\% & 13.1 & 1.3 & 11.5 & 0.7 & 11.4 & 0.6 & 0.001 \\
BEE 40\% & 17.2 & 0.8 & 17.2 & 0.7 & 17.0 & 0.6 & 0.260 \\
BEE 60\% & 19.1 & 1.0 & 18.8 & 0.7 & 18.5 & 0.6 & 0.001 \\
BEE 100\% & 21.4 & 1.3 & 20.8 & 0.8 & 20.1 & 0.8 & 0.001 \\
Nystatin & 26.0 & 0.0 & 26.0 & 0.0 & 26.0 & 0.0 & \\
$p$ & 0.001 & & 0.001 & & 0.001 & & \\
\hline
\end{tabular}

All inhibition halos were measured in millimeters

A level of significance was established at $p<0.05$

$\mathrm{BEE}$, Bixa orellana (L) ethanol extract

for the behavior of said results. The measurements were evaluated by the nonparametric tests of Friedman and Kruskall-Wallis for dependent and independent groups, respectively. Both tests were worked with a level of significance of $p<0.05$.

\section{Results}

Figure 3 shows that when evaluating BEE's antifungal activity, our results showed that the highest activities were present at 24 hours at the concentrations of Nystatin $26 \pm 0.0 \mathrm{~mm}$ and BEE $100 \% 21.4 \pm$ $1.3 \mathrm{~mm}$; however, this activity decreased directly proportional to the time of 48 and 72 hours. On the other hand, the lowest antifungal activity at 24 hours of evaluation was evidenced in the $5 \%$ BEE group $(11.0 \pm 0.0 \mathrm{~mm})$, with no change observed in this group during the three times evaluated 24,48 , and 72 hours (Table 1 ).

Figure 3 shows that when performing the inferential statistical analysis, it was found that at 24, 48, and 72 hours of culture, there were statistically significant differences between nystatin concentrations $(26.0 \pm 0.0 \mathrm{~mm})$ and the six concentrations of

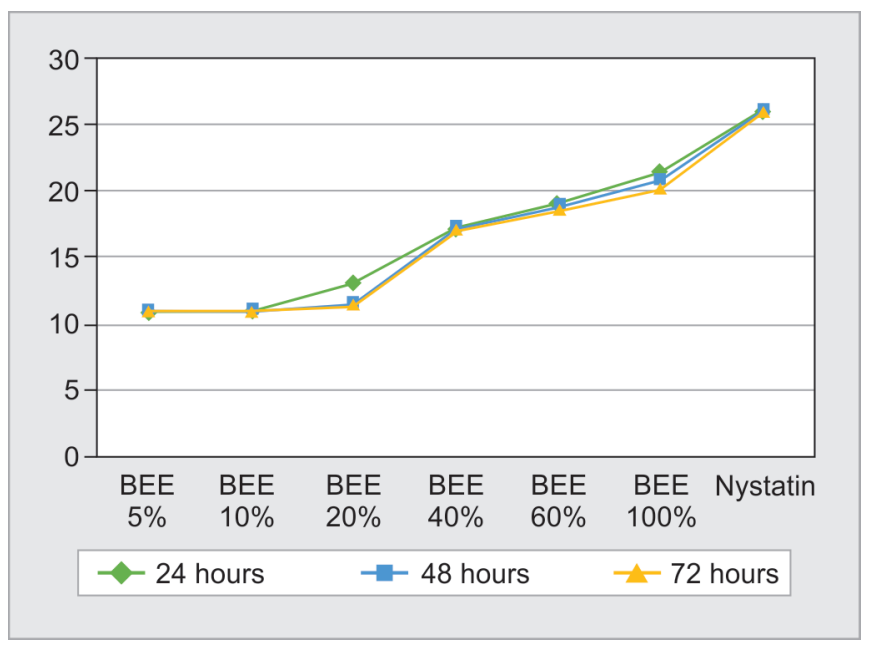

Fig. 3: A comparison of the antifungal activity of $\mathrm{BEE}, \mathrm{BEE}$ : Bixa orellana (L) ethanol extract 
Bixa orellana (L) evaluated (5\%, 10\%, 20\%, 40\%, $60 \%$ and $100 \%)$, with a $p<0.001$. However, when comparing the antifungal activities of the six concentrations of $B E E$, only statistically significant differences were demonstrated at 24, 48, and 72 hours between the concentrations of Bixa orellana $(\mathrm{L})$ at $20 \%(13.1 \pm 1.3),(11.5 \pm 0.7)$, and $(11.4 \pm 0.6)$ respectively with a $p<0.001$. Similarly, it occurred in $60 \%$ and $100 \%$ BEE groups with $p<0.001$ (Table 1).

\section{Discussion}

Bixa orellana (L) is a plant native to South America; its scientific name arose in honor of the Spanish conqueror Francisco Orellana, "Achiote" is one of its main names with which it is known as this natural resource. It is usually a small tree and whose fruit is mainly covered with hairs, containing within deep-red triangular seeds. Currently, its use has been popularized considerably owing to its multiple healing properties: reduction of inflammation, regulation of blood pressure and cholesterol. ${ }^{5,8,9}$ The present investigation determined the antifungal activity of BEE (Achiote) against Candida albicans, using the Kirby-Bauer method or disk diffusion. It was determined that BEE had an inhibitory effect in vitro. According to the Friedman test, it was shown that Nystatin at 100,000 IU/mL had a significantly higher inhibitory effect than the Bixa orellana (L) extract (achiote) $(p<0.05)$ similar to its different concentrations of $5 \%, 10 \%, 20 \%, 40 \%, 60 \%$ and $100 \%$, compared to the Candida albicans at 24, 48 and 72 hours, by measuring the susceptibility rings according to the Duraffourd scale.

In other studies such as those described by Reinhardt, Castello, Medina-Flores, and Castillo, ${ }^{4,8,9,13}$ the antifungal activity against Candida albicans ATCC 10231 was evaluated by agar diffusion and agar dilution methods for the determination of MIC. Of the 12 investigated extracts, 6 presented a consistent antifungal activity, with an inhibition halos diameter $>18 \mathrm{~mm}$ in $100 \%$ concentration (100 mg/mL). From this, it can be deduced that both the average inhibition halos and the MIC were similar to the results of the study.

For instance, according to some studies as described by Ferrerira, Castillo, and Garcia del Prado, ${ }^{12-14}$ it was determined that there is a statistically significant difference in the antifungal effect in the different concentrations of extract of Origanum vulgare on the growth of Candida albicans and the four concentrations were sensitive when comparing the inhibition zones according to the Duraffourd scale. In order to demonstrate the antifungal effect that natural resources may have on treatments in patients with subprosthetic stomatitis, research works have been carried out. ${ }^{12-14}$ On the other hand, there are works (e.g., Velazco et al. ${ }^{15}$ ) showing, by observation and comparison of both samples, the presence of pseudohyphae and chlamydospores in the first sample, including hyphae penetrating towards the defects of the structure inherent to the elaboration process. In the second sample, there was a marked topographic differentiation. This information agrees with the results of the present study, where the methanol extract at higher concentrations ( $75 \%$ and $100 \%$ ) of the leaves of Bixa orellana (L) showed a high antifungal effect at 24 hours and 48 hours as did chlorhexidine $0.12 \%$. Therefore, those concentrations are effective in reducing Candida albicans.

The antimicrobial action of this plant is possibly due to the different chemical compounds it harbors: pectin, carotenoids, petoses, etc. Microscopic evidence demonstrated candidiasis adhesion in the denture in use. ${ }^{16}$ Therefore, there are indications of the usefulness of these natural extracts for the treatment of certain stomatological infections, but these must still be clinically validated with trials performed in accordance with good clinical practices, before any application in the Cuban stomatological services. Also, oral hygiene habits in patients with prostheses are the main methods to prevent the onset of pathology. Once detected, the infection can control the patient with hygienic measures and antifungal agents. It is worth mentioning that Lee et al. ${ }^{17}$ indicated the diagnosis of prosthetic stomatitis in $55.9 \%$ of the subjects, of which type I $=29.4 \%$ and type $I I=26.5 \%$. Candida spp. counts were higher in those with prosthetic stomatitis, both before and after treatment. When installing functional prostheses, the count decreased significantly, however, it remained high in those with prosthetic stomatitis diagnosed prior to the rehabilitation treatment. The most frequently identified species was Candida albicans. The most frequent location of the hypha was the middle and posterior part of the palate. Continuous use of prostheses prevailed as a harmful habit, followed by poor oral hygiene. Acrylic bases were the ones that produced the condition most frequently. The risk of suffering from the disease was increased. In this regard, not all patients with sub-prosthetic stomatitis have Candida albicans, but Candidiasis was increased in the advanced degrees of subprosthetic stomatitis. ${ }^{18-20}$

The main limitation of the study was that due to the feasibility of the study, it was only possible to deal with Candida albicans strains; so it would be important to verify and show that it also has an antifungal potential against other fungi in the oral cavity. However, this research recommends carrying out more in vitro studies that allow measuring the effect of the Bixa orellana (L) (Achiote) plant on the various fungal strains associated with the oral cavity. In addition, more research needs to be conducted on the inhibitory effect of the plant extract against other bacteria present in the oral cavity treatments in order to guarantee its use as a broad-spectrum antifungal agent. Finally, we suggest promoting scientific research on medicinal plants that contribute new knowledge to dentistry and the treatment and elimination of Candida albicans prosthetic be extended.

\section{CONCLUSION}

According to the results obtained in this investigation, it is concluded that among the antifungal activity of the six concentrations of BEE, only statistically significant differences were demonstrated at 24, 48 , and 72 hours between the concentrations of Bixa orellana $(\mathrm{L})$, $20 \%, 60 \%$ and $100 \%$ with $p<0.001$.

\section{ACKnOWLedgments}

The authors thank the School of Dentistry of Universidad Cientifica del Sur, Universidad Nacional Federico Villarreal and the Universidad Nacional Mayor de San Marcos for their support in the execution and development of this research work; also for the unconditional support in help writing and preparing this manuscript.

\section{References}

1. Wilson J. The aetiology, diagnosis and management of denture stomatitis. Brit Dent J 1999;18(8):380-384. DOI: 10.1038/sj.bdj. 4809821.

2. Velazco G, Ortiz R, et al. Microscopic evidence of Candida albicans present in prosthetic bases removed of buccal cavity. Rev Cubana Estomatol 2013;46(2):1-8.

3. Pires FR, Santos EB, et al. Denture stomatitis and salivary Cándida in Brazilian edentulous patients. J Oral Rehabil 2002;29(11):1115-1119. DOI: 10.1046/j.1365-2842.2002.00947.x. 
4. Reinhardt LC, Nascente PS, et al. Sensitivity to antifungals by Candida spp samples isolated from cases of chronic atrophic candidiasis (CAC). Braz J Biol 2019;1:1-7. DOI: 10.1590/1519-6984. 190454.

5. Quiroz JQ, Torres AC, et al. Optimization of the MicrowaveAssisted Extraction Process of Bioactive Compounds from Annatto Seeds (Bixa orellana L.). Antioxidants (Basel) 2019;8(2): 37-47.

6. Soto AF, Mendes EM, et al. Antimicrobial effect and cytotoxic activity of vinegar-hydrogen peroxide mixture: A possible alternative for denture disinfection. J Prosthet Dent 2019;121(6):966.e1-966.e6. DOI: 10.1016/j.prosdent.2019.02.019.

7. Lee X, Vergara C, et al. Severity of Candida-associated denture stomatitis is improved in institutionalized elders who consume Lactobacillus rhamnosus SP1. Aust Dent J 2019. DOI: 10.1111/adj. 12692.

8. Castello M, Phatak A, et al. Antimicrobial activity of crude extracts from plants parts and corresponding calli of Bixa Orellana L. Indian $J$ Exp Biol 2002;40(12):1378-1381.

9. Medina-Flores D, Ulloa-Urizar G, et al. Antibacterial activity of Bixa orellana L. (achiote) against Streptococcus mutans and Streptococcus sanguinis. Asian Pac J Trop Biomed 2016;6(5):400-403. DOI: 10.1016/ j.apjtb.2016.03.005.

10. Calderon A, Salas J, et al. Assessment of Antibacterial and Antifungal Properties and In Vivo Cytotoxicity of Peruvian Passiflora Mollisima. J Contemp Dent Pract 2019;20(2):145-151. DOI: 10.5005/ jp-journals-10024-2489.

11. Mayta-Tovalino F, Gamboa E, et al. Development and Formulation of the Experimental Dentifrice Based on Passiflora mollissima (Tumbo) with and without Fluoride Anion: Antibacterial Activity on Seven Antimicrobial Strains. Int J Dent 2019; 9056590. DOI: 10.1155/2019/9056590.
12. Ferreira F, Silva A, et al. Candida spp. in Complete Dentures of Institutionalized Elderly Individuals. Int J Odontostomat 2016;10(2):283-286. DOI: 10.4067/S0718-381X2016000200015.

13. Castillo D, Tello M, et al. In vitro Susceptibility of Candida albicans and Non-albicans Isolated from Dental Prothesis in Patients with Denture Stomatitis to Three Disinfectants. Int J Odontostomat 2015;9(3):373-377. DOI: 10.4067/S0718-381X2015000300004.

14. García del Prado G, Gutiérrez M, et al. Bixa orellana L: a potential substance for detection of dentobacterial plaque. Rev Cubana Estomatol 2014;20(2):23-31.

15. Velazco G, Ortiz R, et al. Microscopic evidence of Candida albicans present in prosthetic bases removed of buccal cavity. Rev Cubana Estomatol 2013;46(2):67-71.

16. Lourido C, Martínez G. The Bixa orellana L. in treatment of stomatology affections: a subject that hasn't studied yet. Rev Cubana Farm 2013;44(2):231-244.

17. LeeX, Cajas N, et al. Occurrence of genus Candida yeast and prosthetic stomatitis before and after rehabilitation treatment based on removable prosthesis. Rev Clin Periodoncia Implantol Rehabil Oral 2015;8(1):31-37. DOI: 10.1016/j.piro.2015.02.005.

18. Mousavi SA, Ghotaslou R, et al. Evaluation of antibacterial and antifungal properties of a tissue conditioner used in complete dentures after incorporation of $\mathrm{ZnO}-\mathrm{Ag}$ nanoparticles. J Dent Res Dent Clin Dent Prospects 2019;13(1):11-18. DOI: 10.15171/ joddd.2019.002.

19. Fleischer TC, Ameade EP, et al. Antimicrobial activity of the leaves and seeds of Bixa orellana. Fitoterapia 2003;74(1-2):136-138. DOI: 10.1016/S0367-326X(02)00289-7.

20. Rojas JJ, Ochoa VJ, et al. Screening for antimicrobial activity of ten medicinal plants used in Colombian folkloric medicine: a possible alternative in the treatment of non-nosocomial infections. BMC Complement Altern Med 2006;6:2. DOI: 10.1186/1472-6882-6-2. 